\title{
Ф-OTDR Distributed Fiber Optic Vibration Signal Denoising Method Based-on Wavelet Packet
}

\author{
Zhang Jian ${ }^{1,2} *$ Li Wei $^{2}$ and $\mathrm{Li} \mathrm{Wei}^{3}$ \\ ${ }^{1}$ College of Computer Science, Yangtze University, Hubei JingZhou, 434023 \\ ${ }^{2}$ National Engineering Laboratory of Fiber Optic Sensing Technology, Wuhan \\ University of Technology, Wuhan, 430070 China \\ ${ }^{3}$ School of Computer Science and Technology, Wuhan University of Technology, \\ HuBei Wuhan, 430070 China \\ 'zhangjian0716@126.com, ${ }^{2}$ limighty@126.com, 3 lwei027@163.com
}

\begin{abstract}
An optimized denoising technique based on wavelet packet is proposed to address the defects of traditional phase-sensitive optical time domain reflectometer ( $\Phi-O T D R$ ) based distributed vibration sensor systems, which are known for unsatisfactory denoising performance when engaging in external vibration signal denoising and comparing respective spectrum. The signals acquired from vibration points were analyzed in the first place to identify their features and an optimal fundamental wave Sym6 was selected. The number of fundamental wave decomposition layers was investigated for its effect on raw signal denoising performance, three layers being retained as the optimum layer number. Secondly, the pre-processed and post-processed signals were treated using Fourier transformation technique, by means of frequency comparison, wavelet packet denoising technique has been found effective in removing noises other than the master vibration signal. A frequency spectrum analysis performed with respect to the sampling points neighboring the vibration points found that only the sampling points corresponding to vibrations points had a frequency identical to the vibration frequency, therefore demonstrating that the system may provide an actual spatial resolution up to $1 \mathrm{~m}$ level.
\end{abstract}

Keywords: Wavelet Packet, Phase-sensitive Optical Time Domain Reflectometer $\left.{ }^{\phi-\mathrm{OTDR}}\right)$, Distributed Vibration Sensing ; Fourier transform;

\section{Introduction}

The distributed optical fiber sensing system based on phase sensitive optical time domain reflectometer technology in the security area is not only anti-electromagnetic interference, corrosion resistance, high sensitivity, and has wide field about distance monitoring ability, long-distance positioning capability, and other prominent advantages. Therefore, the system plays an important role at intrusion detection and security that relevant to the border, military bases, long pipelines, long transmission lines, long distance communications cable and other important application areas as well.

Due to The $\phi-\mathrm{OTDR}$ technology has great application prospect in the perimeter security field, domestic and foreign researchers did plenty of study around the technology deeply through the past 20 years [1-3]. K.N.Choi et applied $\phi-O T D R_{\text {technology to the }}$ Ground Intrusion detection system based on distributed optical fiber sensor in 2003, however, the positioning accuracy is only $1 \mathrm{~km}$ and positioning range is about $12 \mathrm{~km}$, the SNR is only about 5.6dB in the field experiment[4]. Most of studied jobs that had been reported relevant to the vibration sensor based on $\phi-\mathrm{OTDR}$ were focus on improving the spatial resolution and the sensing distance, but the contents about disturbance 
frequency detection were reported rarely [5-9]. Lu et al put forward a kind of coherent detection method based on $\phi-\mathrm{OTDR}$ to measure the disturbance frequency of external acoustic field in 2010, they obtained disturbance frequency that using fast Fourier transform way to process the original datas, in addition, they reduced the noise using moving average method, and the system measurement bandwidth were also be reduced [6]; Muanenda detected external acoustic field oscillation frequency through the $\phi-\mathrm{OTDR}$ system that consisted of the distributed feedback laser, the maximum measurement frequency can be reached is $500 \mathrm{~Hz}$, positioning range is $5 \mathrm{KM}$, accuracy is about 5m [10]; Martins, HF et al expanded $\phi-\mathrm{OTDR}$ system's positioning range to $125 \mathrm{KM}$, otherwise the resolution is only $10 \mathrm{~m}$, due to the limitation of light pulse which can only measure the disturbance frequency less than $380 \mathrm{~Hz}$ [11]. Since the effective Signal of the system is too weak and affected by environmental noise pollution, therefore resulting in a poor SNR.

In order to improve positioning accuracy and sensitivity for sensing the weak vibration signal. researchers process the system signal using pre-denoise method generally, the moving average [12] and wavelet threshold denoising method use widely as the two mainstream ways[13]. Moving average method is generally that making the current backward Rayleigh scattering signal sub the previous backward Rayleigh scattering signal in a row, the computing workload of this kind of algorithm is enormous and the external environment changes will cause error happen. on the other hand, Wavelet threshold denoising method has a very good ability to distinguish noise and signal, but due to the lack of translation invariance, the resulting denoising signal always be distort.

This paper presents a denoising optimization method based on wavelet packet. Firstly, vibration point signal is denoised by wavelet packet analysis way in order to overcome the defects of large amount of computation workload that using the moving average method and signal distortion that using wavelet denoising method. Secondly, the system can obtain accurate vibration frequency information from the denoised signal that processed by Fourier transform, it proved that the wavelet packet analysis denoising method is effective; thirdly, While the spectrum analysis of the collection point near the vibration point show that only the vibration frequency and vibration frequency of collection points corresponding to the vibration point are the same, in conclusion, it proved that actual spatial resolution of the system is up to $1 \mathrm{~m}$.

\section{Measuring Principle}

\subsection{Vibration Signal Characteristics Analysis}

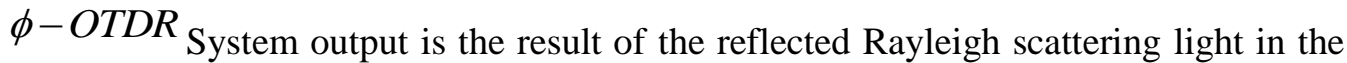
pulse width region coherence interferometry, the interferometry of backward Rayleigh scattering of the fiber in pulse range is approximately as Fabry-Perot interferometer of the fiber, in such conditions, coherent backward cattering light power is formula (1) as follow [14]:

$$
P_{b s}^{\prime}=P_{i n}^{\prime} 2 R(1+\cos \phi)
$$

formula (1)

Where: $P_{b s}^{\prime}$ is the backscattered light power after interference; $P^{\prime}{ }^{\prime}$ is the transmission optical power of this point; $R$ is approximately as the backscattering coefficient; $\phi_{\text {is the }}$ retardation between light pulse rising and falling .

When the external vibration is applied to the sensing fiber, optical phase changes within the pulse region, the formula (1) show that the backscattered light power of vibration position change, thus External vibration can be detected through the change of 
the probe light power. The relationship between the pulse width $\mathrm{W}$ and the system resolution $\Delta Z$ as follows:

$$
\Delta Z=W V / 2 \quad \text { formula }
$$

In addition, Energy loss is unavoidable in optical fiber transmission, so the proximal end intensity of rayleigh scattering light is strong, the distal end intensity is weak, the noise remains the same, since the collected data is the original vibration signal located in the fiber end, furthermore, it need to denoise the original signal before the original data processed by fourier transform way in order to improve the SNR. The system noise mainly comes from the optical path, circuit and the external environment. The noise of optical path could be improved and suppressed by selecting and adjusting the optical path, so the denoising processing is aim at noise caused by circuit and external environmental. According to the characteristics of vibration signals in the system, the wavelet packet denoising method that be selected as a most appropriate method using in the system. [15].

\subsection{Wavelet Packet Denoising Method Principle}

Wavelet packet filtering denoising method is similar to wavelet analysis method, the principle of signal filtering denoising can be explained from the perspective of wavelet multi-resolution, except that the wavelet packet filtering method is more complex and flexible than the others. figure $\mathbf{1}$ is a schematic diagram that indicated wavelet decomposition, 3 layer wavelet packet decomposition (layer 3 as an example) of signal $\mathrm{S}$ respectively, A represents the low-frequency approximate section, D represents the detail about high frequency section, the End Number represents the layer number of the wavelet decomposition (i.e. scale number). As can be seen from the chart, the wavelet packet decomposition method can divide signal frequency band by the multi-level way, and match any signal spectrum adaptively according to the signal characteristics and practical analysis requirements, and can analysis the signal spectrum accuratlier than before.

Signal decomposition is expressed as follows: (3 layers as an example)

$$
s(t)=A A A 3+D A A 3+A D A 3+D D A 3+A A D 3+D A D 3+A D D 3+D D D 3 \text { formula }
$$

Wavelet coefficients that after wavelet transform process indicate the similarity degree between the wavelet and the signal that be processed, if the wavelet and the original signal waveform is more similar, the reconstruction error is smaller.

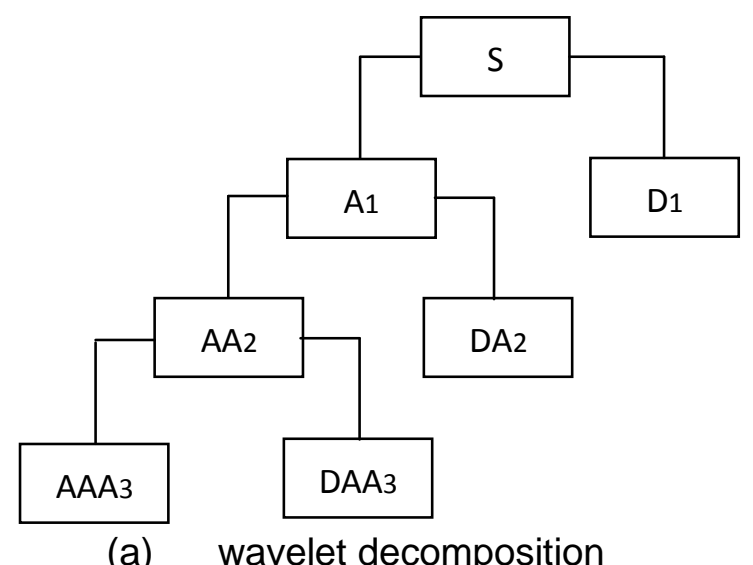

(a) wavelet decomposition 


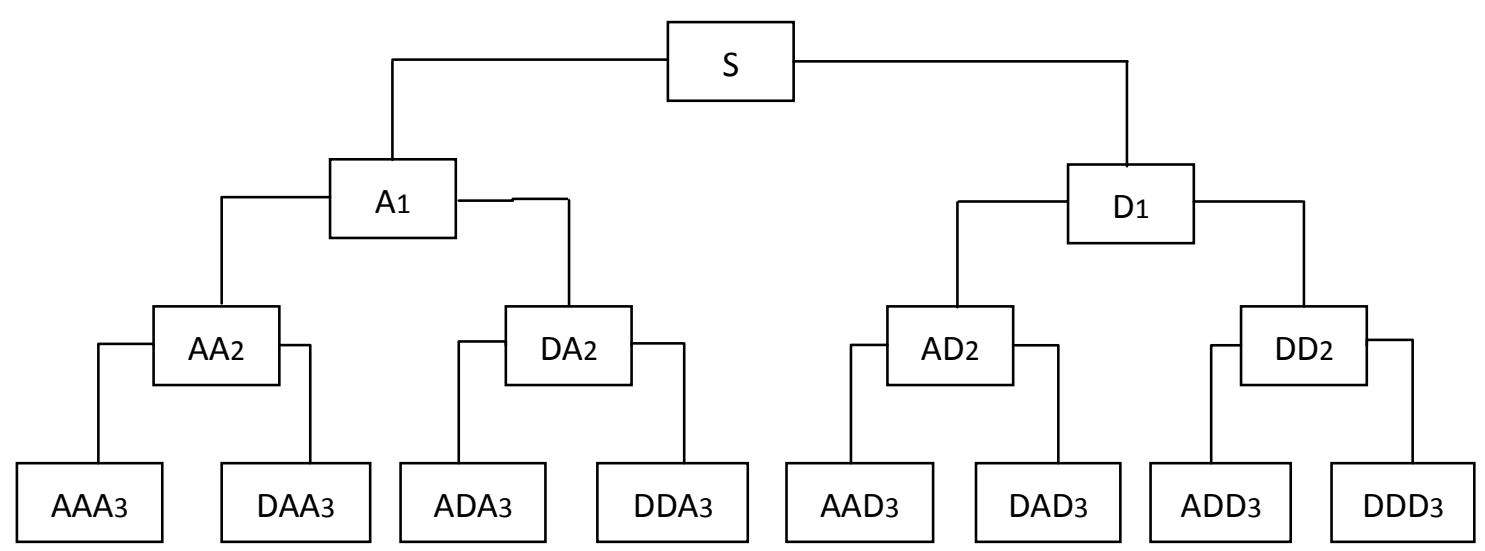

(b) wavelet packet decomposition

Figure 1. Two Kinds of Wavelet Decomposition Diagram

Compared with wavelet analysis, wavelet packet analysis method is a more sophisticated multi-resolution analysis way. Furthermore. It divides signal frequency band with multi-layer in full-band scale, and decompose the high-frequency section that no breakdown wavelet analysis signal. Decompose signal using wavelet packet method, According to the research target frequency characteristics, the wavelet packet coefficients in the different characteristic bands are reconstructed in order to eliminate the noise located external characteristic band position.

The wavelet packet filtering process that aim at the phi-OTDR distributed optical fiber vibration signal is as follows :

Step 1: Acquire raw data of optical fiber vibration signal that containing noise in phi-OTDR distributed optical fiber sensing system, and select optical wavelet function while determine wavelet packet decomposition layers according to the data characteristics of the vibration signal and a large number of tests and measures;

Step 2: the original data are filtered by Wavelet packet decomposition way ,and acquire a plurality of corresponding wavelet packet coefficients according to the frequency band from low to high:

the original data are Multiple iterated and decomposed with a first wavelet filter $\mathrm{H}(\mathrm{x})$ and a second wavelet filter $\mathrm{G}(\mathrm{x})$ respectively, as while fast Fourier transform and interval sample each iteration decomposition results until the number of layers is under required, to acquire the coefficient responding to each original data band; Specific decomposition formula is as follows:

$$
\left\{\begin{array}{l}
P_{0}^{1}(t)=f(t) \\
X_{j}^{2 i-1}(k)=\sum_{n=0}^{N_{j}-1}\left[\sum_{k} H(k-2 t) P_{j-1}^{i}(t)\right] w_{N}^{k n}, w_{N}=e^{-j \frac{2 \pi}{N}}, 0 \leq k \leq \frac{N_{j}}{4} \cup \frac{3 N_{j}}{4} \leq k \leq N_{j} \\
X_{j}^{2 i}(k)=\sum_{n=0}^{N_{j}-1}\left[\sum_{k} G(k-2 t) P_{j-1}^{i}(t)\right] w_{N}^{k n}, w_{N}=e^{-j \frac{2 \pi}{N}}, \frac{N_{j}}{4} \leq k \leq \frac{3 N_{j}}{4}
\end{array}\right.
$$

Formula(4)

Therein, $f(t)$ is the original data, $t$ is discrete time series $; \underline{t=\left\{2^{0}, 2^{1}, \ldots, 2^{j}\right\}}$; $i=\left\{2^{0}, 2^{1}, \ldots, 2^{j}\right\} 。 j$ is layer number ; $j=\log _{2} N-1 ; N$ is the wavelet function vanishing moments, also data points of layer $j$ after decomposition;

Step 3: Select soft or hard threshold function to quantize each band coefficient 
obtained from the wavelet packet decomposition process, and set the spectrum value of excess frequency components in the spectrum as zero;

Step 4: Use the best wavelet packet basis decomposition coefficients and wavelet packet coefficients after threshold to reconstruct wavelet packet quickly, as follows :

Firstly, insert interval zero into wavelet packet coefficients for each band, and then reconstruct data using the first wavelet filter $\mathrm{H}$ (x) or the second wavelet filter $\mathrm{G}(\mathrm{x})$, finally, do fast Fourier inverse transform to reconstruct fast wavelet packet through several iterations of the above procedure;

Reconstruction formula is as follows:

$$
\begin{aligned}
& X_{j}^{i}(k)=2\left\{\sum_{n=0}^{N_{j}-1}\left[\sum_{k} H(t-2 k) P_{j+1}^{2 i-1}(t)\right] w_{N}^{k n}+\sum_{n=0}^{N_{j}-1}\left[\sum_{k} G(t-2 k) P_{j+1}^{2 i}(t)\right] w_{N}^{k n}\right\} ; \\
& w_{N}=e^{-j \frac{2 \pi}{N}} ; 0 \leq k \leq N_{j}
\end{aligned}
$$

therein, $j=\log _{2} N-1 ; i=\left\{2^{j}, 2^{j-1}, \ldots, 2,1\right\}$;

Step 5: Compare features main peak height, peak position extracted from the vibration signal and peak height, peak position before filter, if it meets the requirements, then save the light vibration signal after filtered; otherwise jump to step 3, until it meets the requirements.

When it need to extract a certain frequency signal component or some specific frequency signal components from complex mixed signal, there will be exist the big deviation due to serious frequency folding-mixed phenomenon in the wavelet coefficient obtained from the decomposing algorithm, the extra frequency components of each node can be removed using the above Fourier transform and Fourier inverse transform effectively and certain extent improvement with the above algorithm.

\section{The Experimental Design Based on ${ }^{\phi-O T D R}$ Distributed Optical Fiber Sensing System}

Like the conventional $\phi-\mathrm{OTDR}$ system, the $\phi-\mathrm{OTDR}$ system can measure the delay time between the input pulse signal and the received pulse in order to obtain the vibration position. When uncertain position located in fiber optic line occurs vibration as the result of the invasion, optical fiber's refractive index and the length will also change in the corresponding position, this will result in the optical phase changing. Due to the reflection effect, phase change will cause changes of the backscatter intensity light. Since scattered light of the invasion position transmitted to the detector is through periodic phase change, so the final result of the interference will vary, and corresponds to the invasion position. 


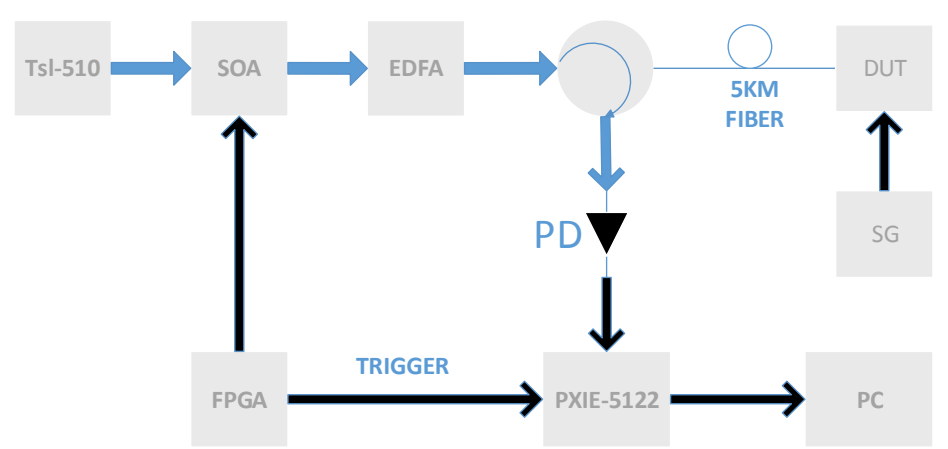

Figure 2. Experimental setup for coherent phase-sensitive OTDR

Experimental design based on $\phi-\mathrm{OTDR}$ distributed optical fiber sensing system is as shown in figure 2, FPGA generates a $10 \mathrm{~nm}$ pulse width and $2 \mathrm{~K}$ cycle periodic pulse signal, the continuous light through SOA is modulated using this signal, generated continuous light that laser emits is modulated into light pulses through SOA, light pulses are amplified through the erbium-doped fiber amplifier (EDFA), and then transmitted into the sensing fiber through a $3 \mathrm{~dB}$ coupler, the backward Rayleigh scattering interference light is detected by a photodetector. The $0.5 \mathrm{~m}$ fiber wound on PZT vibrator at the end of the $5 \mathrm{Km}$ sensing fiber. The signal generator $(\mathrm{SG})$ produce different frequency sine wave to drive this vibrator and use the PXIE-5122 data acquisition card for collecting data with a sampling rate of $100 \mathrm{M} / \mathrm{S}$. Finally, the collected data are processed through Matlab software.

\section{The Experiment Results Analysis}

Applying a $6 \mathrm{~V}$ and $600 \mathrm{~Hz}$ sinusoidal drive signal to vibrator through signal generator in the $5 \mathrm{Km}$ area of the optical fiber, and the vibrator vibrate fiber here. This paragraph extracted several change values over time of several sampling points near the vibration optical fiber, the time length of each sampling point is $4 \mathrm{~s}$, and then the signals are processed by Fourier transform method. figure $\mathbf{3}$ is one graphical representation relevant to the points, in order to see the changes in the graph clearly, the $0.25 \mathrm{~s}$ time length signal is only shown, it can be seen from the figure, the overtime sampling point value variations are similar to sine wave, signal spectrum has a peak near the $600 \mathrm{~Hz}$, and the other sampling points' signal spectrum have not peaks at $600 \mathrm{~Hz}$

The resolution that be calculated through experimental parameters and formula (3), Space theoretical resolution of this system is $1 \mathrm{~m}$, and in this experiment the $0.5 \mathrm{~m}$ fiber wound on PZT vibrator to detect whether the corresponding sampling point's signal is subjected to vibration caused by the vibrator. Through spectrum analysis relevant to the respective sampling points, only the signal spectrum of one sample point has a peak at the corresponding driving frequency, so the actual resolution of the system can be obtained as $1 \mathrm{~m}$. 

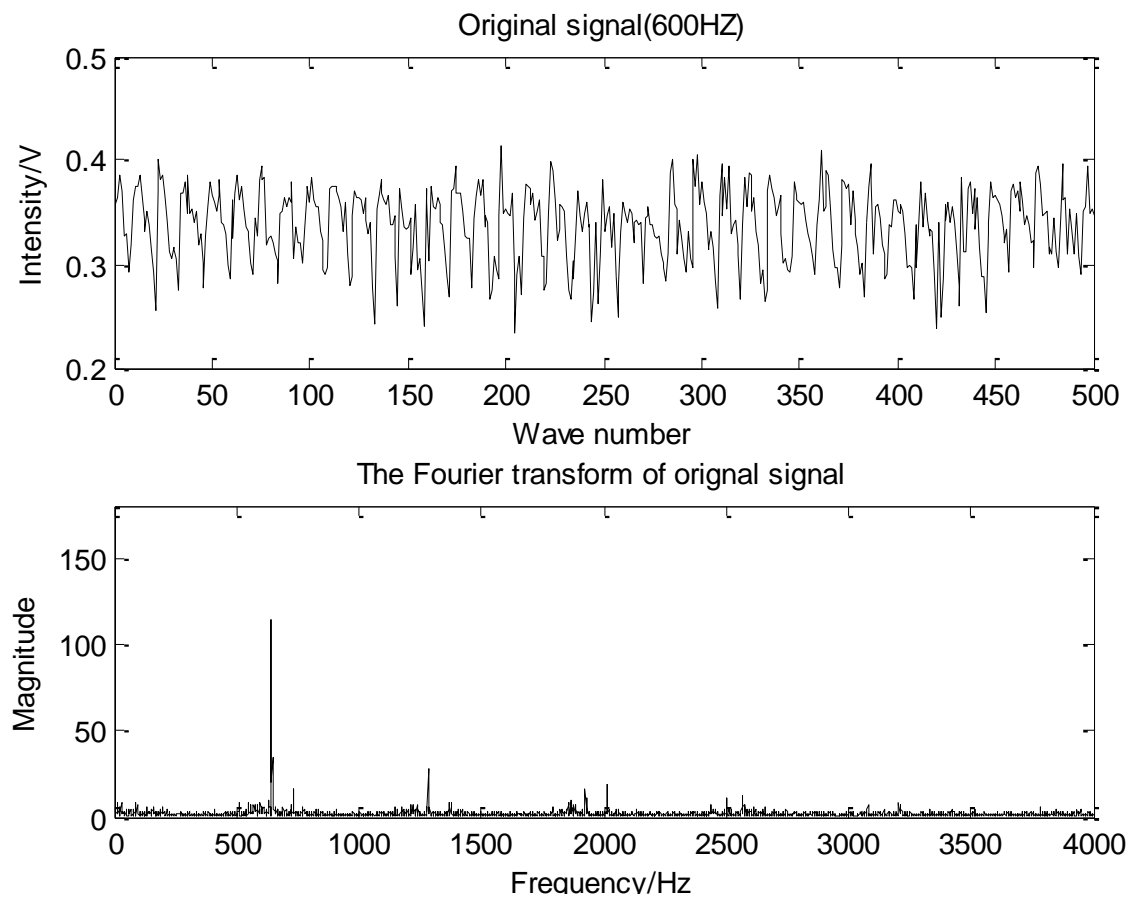

Figure 3. Rayleigh Interference Signal and Spectrum by $600 \mathrm{HZ}$ Sine Drive Signal

As the fundamental wave choice, the fundamental wave with good symmetry does not occur phase mutation situation, excellent regular wavelet is easy to obtain smooth reconstructed signal. According to the characteristics of signal need to be processed, this paper chooses sym6 fundamental wave with excellent symmetry and regularity, and selected 2,3,4,5 level wavelet packet to decompose respectively. The denoised signal is shown as figure 4. As can be seen from the different level denoising decomposition diagrams, with the wavelet packet decomposition level increases, the denoising effect become better, but when the decomposition level increase to three, it is easy to remove the valid signal as noise, finally the decomposition level is setted as three level decomposition 

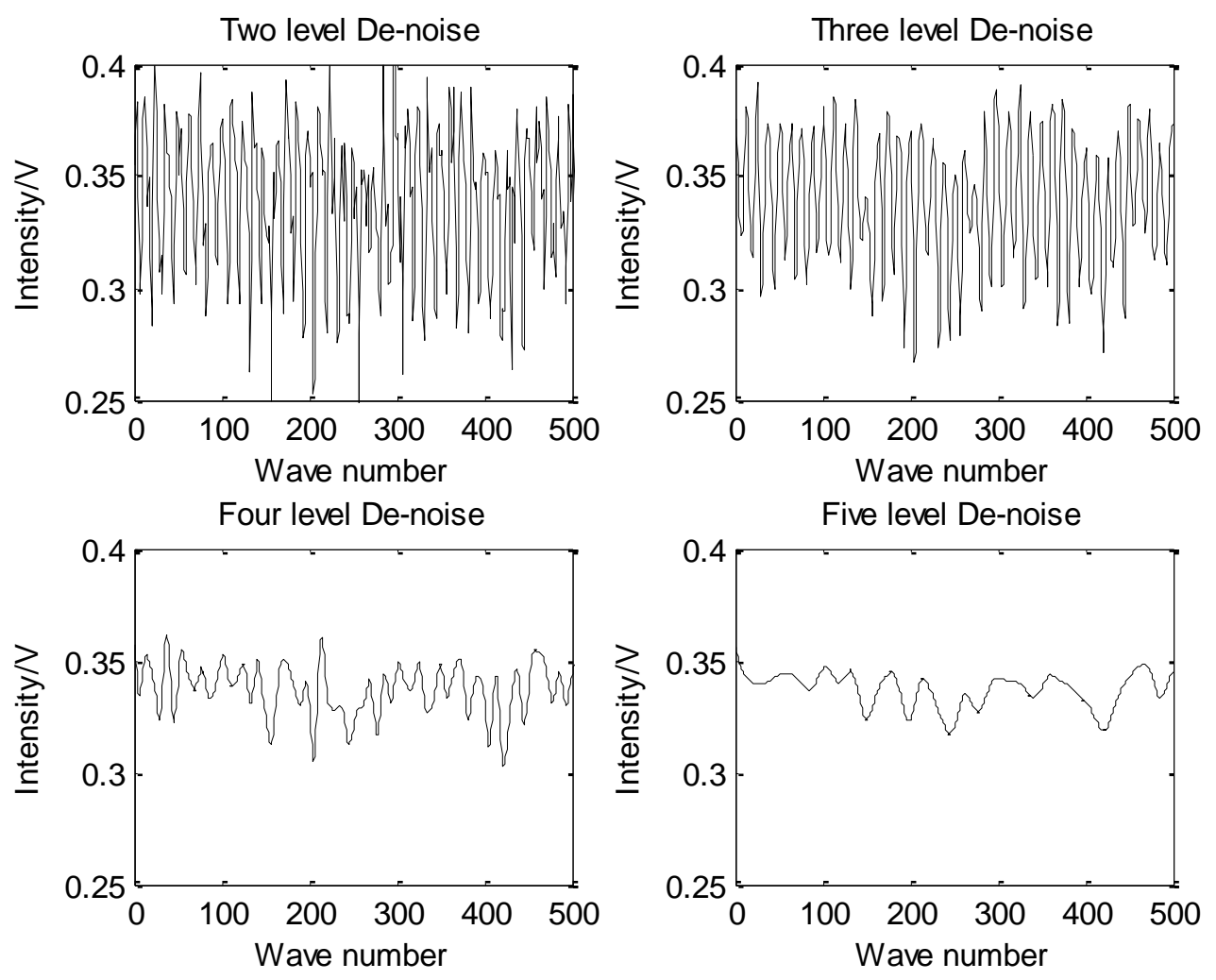

Figure 4. Results using Wavelet Packet Decomposition

Using sine wave signal to drive the vibration sensor with different frequencies respectively, when $600 \mathrm{HZ}$ sine wave appear, signal and spectrum that collected by capture cards as well as the signal and spectrum through the wavelet denoising process are shown in figure 5, the time length of collected data is $4 \mathrm{~s}$ and in order to see the details of the graph only $0.25 \mathrm{~s}$ is shown. As can be seen from the figure, the original signals have higher amplitude components in the low frequency range, the reason is that there is presence of a DC offset in the signals and the DC offset component is greater than the signal original amplitude, which can be processed through the DC filter way later. At the same time, there is a peak at the integral multiple of $600 \mathrm{~Hz}$ due to the vibration frequency doubling phenomenon. The signal after the wavelet packet analysis denoising process is smooth, the spectrum component in high-frequency is zero substantially, there is a peak near the $600 \mathrm{HZ}$ frequency position, this peak value is equal to the peak value before denoising, which is proved that the wavelet packet analysis method retains the useful part of signals and can remove the noise in the signals effectively. When the driving signal is $900 \mathrm{~Hz}$ sine wave, Figure 6 is the signal that capture cards collected and spectrum as well as the signal and spectrum after the wavelet packet denoising, and the comparison results are the same as figure 6 present, it prove that the wavelet packet analysis denoising method is effective than the others. 

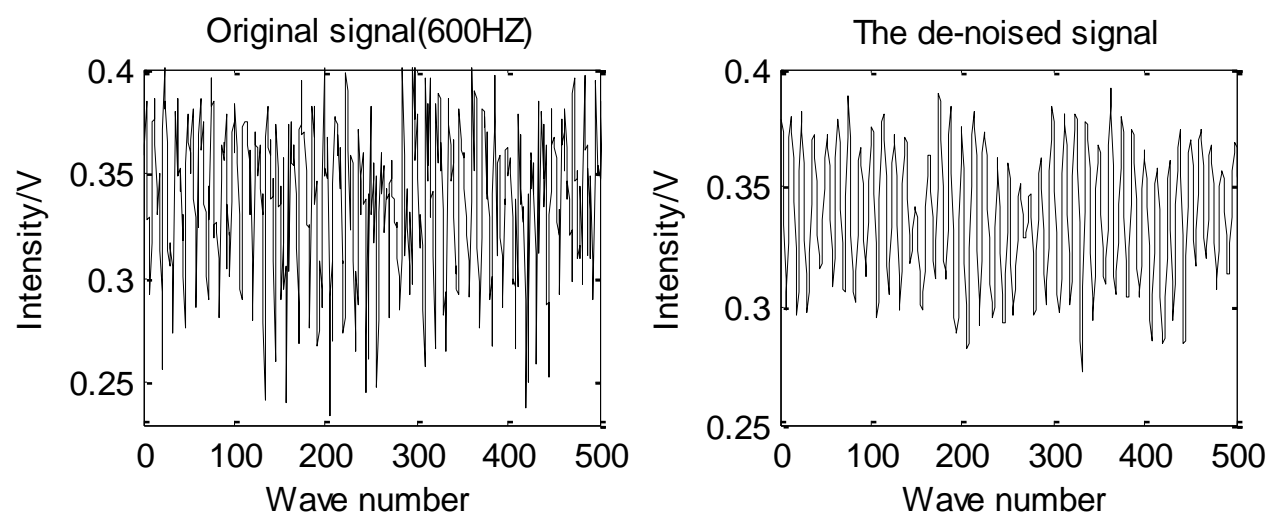

The Fourier transform of orignal signal

The Fourier transform of de-noised signal
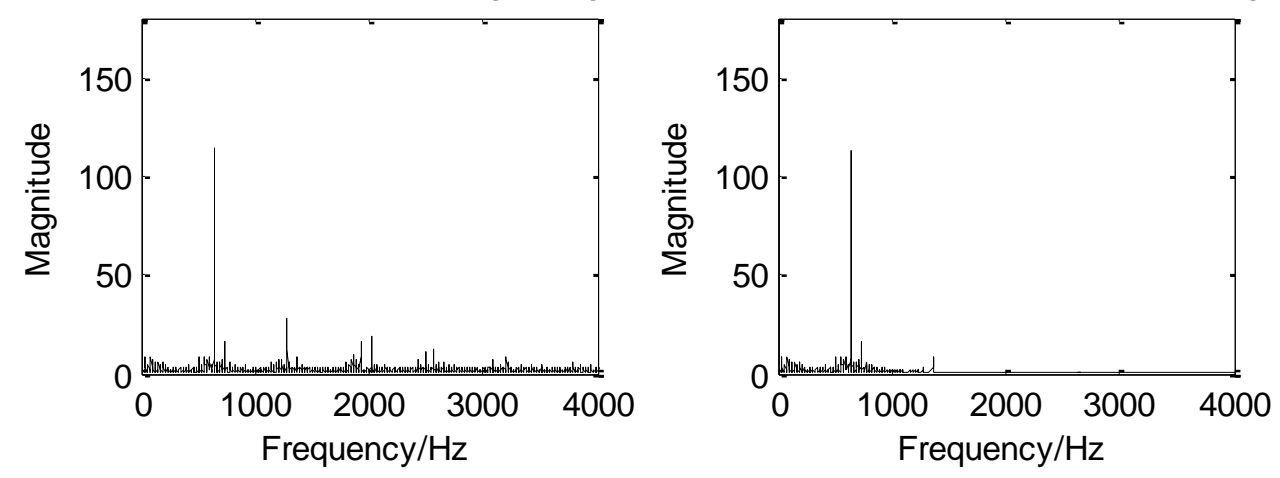

Figure 5. Denoising and No-denoising of the Rayleigh Interference Signal and Spectrum by $600 \mathrm{HZ}$ sine Drive Signal
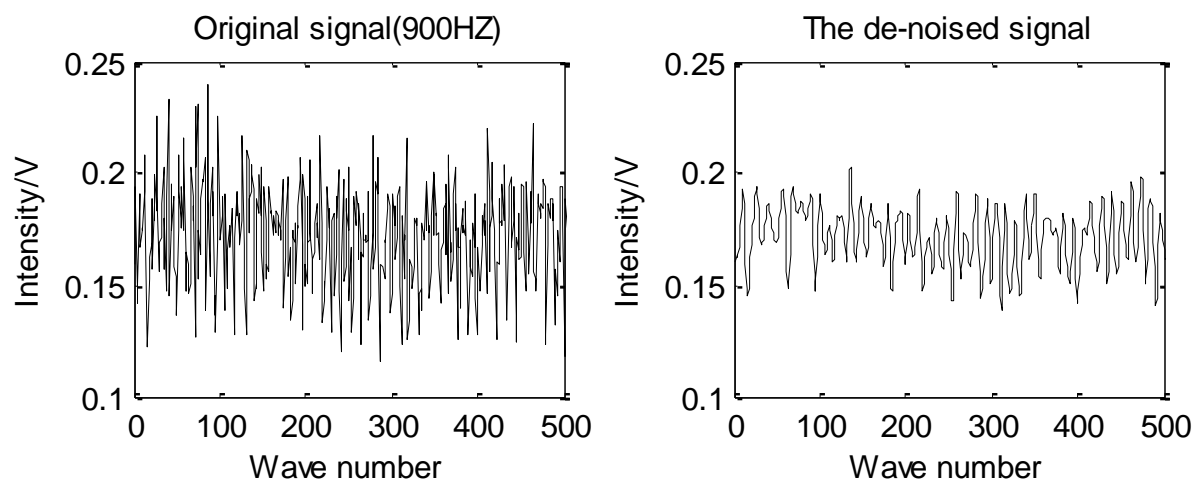

The Fourier transform of orignal signal

The Fourier transform of de-noised signal
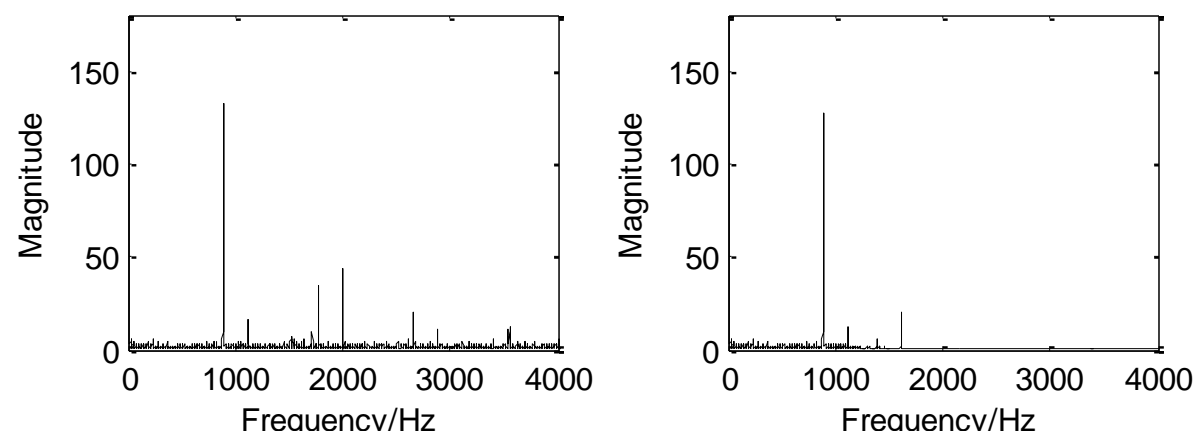

Figure 6. Denoising and No-denoising of the Rayleigh Interference Signal and Spectrum by $900 \mathrm{HZ}$ Sine Drive Signal 


\section{Conclusion}

This paper analyzes the effective signal characteristics and the noise source based on the $\phi-\mathrm{OTDR}$ distributed optical fiber vibration sensing system and preprocess the signal using wavelet packet analysis method, choose the optimal base package according to signal and noise characteristics and denoise the original signal by comparing different wavelet packet decomposition level. The signal that before and after the processing are processed using Fourier transform way, by comparing the respective spectrum, the results prove that wavelet packet analysis denoising method can effectively remove the noise beyond vibration signal. Through the spectrum analysis relevant to the collection point near the vibration point that only the frequency and vibration frequency of collection points corresponding to the vibration point are the same, this kind of situation prove that the system's actual spatial resolution is up to $1 \mathrm{~m}$.

\section{Acknowledgements}

This work was supported by the National Natural Science Foundation of China under Grant No. 61290311, and Educational Commission of Hubei Province of China under Grant No. B2015445,

Humanities and Social Science Fund of Yangtze University under Grant No. 2015csy002,

\section{References}

[1] H. F. Taylor and C. E. Lee, "Apparatus and method for fiber optic intrusion sensing”, U.S. Patent. vol. 5, (1993), pp. 194-847.

[2] J. Park, W. Lee and H. F. Taylor, "A fiber optic intrusion sensor with the configuration of an optical time domain reflectometer using coherent interference of Rayleigh backscattering", Proceedings of SPIE, (1998), 3555, pp. 5-49.

[3] K. N. Choi and H. F. Taylor, "Spectrally stable Er: fiber laser for application in phase-sensitive optical time-domain reflectometry", IEEE Photon. Technol. Lett., (2003), 15(3): 386-389.

[4] Juarez J C,Maier E W ,Kyoo Nam Choi, Taylor H F. Distributed fiber-optic Intrusion sensor system [J], Journal of Lightwave Technology, (2005), 23: 208- 2081.

[5] Ren, M.,Lu, P.,Chen, L., Theoretical and Experimental Analysis of $\Phi$-OTDR Based on Polarization Diversity Detection[J], IEEE Photonics Technology Letters,(2016),28(6):697-700.

[6] Y.Lu, T.Zhu, Distributed vibration sensor based on coherent detection of phase-OTDR[J].J.Lightwave Technol, (2010),28(22):3243-3248.

[7] Martins, Hugo F., Phase-sensitive optical time domain reflectometer assisted by first-order raman amplification for distributed vibration sensing over $>100 \mathrm{~km}[\mathrm{~J}]$, Journal of Lightwave Technology, (2014),32(8):1510-1518.

[8] C.K. Madsen, T. Bae, and T. Snider, Intruder Signature Analysis from a Phase-sensitive DistributedFiber-optic Perimeter Sensor [CA], Proc. of SPIE, (2007), Vol.6770.

[9] Fei Peng, Ning Duan, Yun-Jiang Rao, and Jin Li, Real-Time Position and Speed Monitoringof Trains Using Phase-Sensitive OTDR[J], IEEE PHOTONICS TECHNOLOGY LETTERS, (2014),Vol. 26, NO. 20.

[10] Muanenda, Yonas, Oton, Claudio J., Faralli, Stefano, A Cost-Effective Distributed Acoustic Sensor Using a Commercial Off-the-Shelf DFB Laser and Direct Detection Phase-OTDR[J], IEEE PHOTONICS JOURNAL, (2016),8(1).

[11] Martins, HF, Distributed Vibration Sensing Over $125 \mathrm{~km}$ With Enhanced SNR Using Phi-OTDR Over a URFL Cavity[J], JOURNAL OF LIGHTWAVE TECHNOLOGY, (2015),33(12):2628-2632.

[12] Yang Bin, Gao Wei. Key Technologies for based distributed fiber-optic sensing systems [J].Study on Optical Communications, (2012),172:19-22.

[13] SHANG Jing,YANG De-wei. Wavelet threshold denoising method used in disturbance sensing system [J]. Modern Electronic Technology, China, (2012), 35 (17): 51-53.

[14] Yang Shining .The virtual instrument platform model of distributed optical fiber sensing system [J]. electronic measurement technology, (2012),35 (1): 89-92.

[15] Zhang Defeng. Matlab wavelet analysis and engineering application [M]. Beijing: National Defense Industry Press, (2008). 